\title{
An Optimized CLBP Descriptor Based on a Scalable Block Size for Texture Classification
}

\author{
Jianjun $\mathrm{Li}^{1}$, Susu Fan ${ }^{1}$, Zhihui Wang ${ }^{2}$, Haojie $\mathrm{Li}^{2}$ and Chin-Chen Chang ${ }^{3}$ \\ ${ }^{1}$ School of Computer Science and Technology, Hangzhou Dianzi University, China \\ [e-mail:lijjcan@gmail.com] \\ ${ }^{2}$ School of Software Technology, Dalian University of Technology, China \\ [e-mail:wangzhihui1017@gmail.com] \\ ${ }^{3}$ Department of Information Engineering and Computer Science, Feng Chia University \\ [e-mail:alan3c@gmail.com] \\ *Corresponding author: Jianjun Li
}

Received September 20, 2016; revised November 1, 2016; accepted November 28, 2016; published January 31, 2017

\begin{abstract}
In this paper, we propose an optimized algorithm for texture classification by computing a completed modeling of the local binary pattern (CLBP) instead of the traditional LBP of a scalable block size in an image. First, we show that the CLBP descriptor is a better representative than LBP by extracting more information from an image. Second, the CLBP features of scalable block size of an image has an adaptive capability in representing both gross and detailed features of an image and thus it is suitable for image texture classification. This paper successfully implements a machine learning scheme by applying the CLBP features of a scalable size to the Support Vector Machine (SVM) classifier. The proposed scheme has been evaluated on Outex and CUReT databases, and the evaluation result shows that the proposed approach achieves an improved recognition rate compared to the previous research results.
\end{abstract}

Keywords: Texture classification and recognition; LBP; CLBP; SVM; Scalable block size 


\section{Introduction}

$\mathbf{F}_{\text {eature of texture is one of the basic properties of the surface or structure of an object and }}$ also is a hot research area in computer vision recently. In analysis of texture, classification of texture always has been an important topic of research, and it involves pattern recognition, applied mathematics, statistics, and other research fields. The extraction of the features of texture is a key step in description and classification of texture. Therefore, in the past few decades, extensive research has been conducted on methods of the extraction of features of texture, and many methods have been developed in this field, such as the well-known gray level run length, gray level co-occurrence matrix and self-correlation function. Research of texture feature extraction has been enriched significantly by the continuous expansion of the application field and the introduction of new theories, such as fractal theory, Markov random field theory, and wavelet theory.

Ojala et al. [1] proposed an LBP feature descriptor. Because of its high feature discrimination and low computational complexity, LBP has gained extensive attention in the research community. In analysis of image, computer vision and pattern recognition, LBP have been used extensively. Especially, the use of LBP has been studied and developed extensively in texture classification and face recognition (Ahonen and Hadid [2], Zhao and Pietikanen [3], Nanni and Lumini [4], Shih and Chuang [5]). Because the original LBP is extracted in binary image and achieves binary values by comparing the gray values of the central pixel and its neighbor pixels, it easily can be affected by noise, is sensitive to rotation, and also loses its local spatial structure. Fortunately, many improved LBP descriptors have been proposed and have their own advantages, e.g., dominant LBP [6], LBPV [7], BRINT [8], ELBP [9], FLBP [10], CLBP [11], and CS-LBP [19] which has been widely used [21]. In this paper, we propose a new method that combines the LBP descriptors with scalable block size for texture classification as we call SB-CLBP. The scalable block can keep both the coarse-grained and the detailed information of the texture image. Meanwhile, CLBP takes into consideration the magnitude component and the intensity value of the center pixel to add additional discriminant information. Therefore, the SB-CLBP is able to improve the accuracy of classification of texture.

The remainder of this paper is organized as follows: LBP, multiple LBPs, and CLBP are briefly introduced in Section 2. Section 3 presents the details of SB-CLBP. The experimental results and analysis are addressed in Section 4. Our conclusions are presented in Section 5.

\section{Related Work}

\subsection{Local binary patterns (LBP)}

The LBP descriptor is very simple and has been used extensively for texture analysis. It first was proposed by Ojala et al. to characterize the spatial structure of local image texture. It represents the local texture features of an image by comparing the difference between the gray values of the central point and its neighboring pixels. The original LBP operator defined a window with $3 \times 3$ pixels, and its threshold was the central pixel of the window. All neighbors with values higher than or equal to the value of the central pixel are given a value of 1 , while neighbors with values lower than the value of the central pixel are given a value of 0 . Therefore, an 8-bit binary number can be generated from the comparison of eight neighboring 
points and it is assigned as an LBP value of the central pixel in the window and it reflects the texture information of the region.

In order to adapt to the texture features of different scales, the LBP operator was improved by Ojala et al. They extended the 3x3 size of the neighborhood to any size and used a circular neighborhood instead the square neighborhood. The improved LBP operator is allowed to have any number of pixels in the circular neighborhood with a radius of $\mathrm{R}$, so we can get the LBP operator, such as a circular area with a radius of $\mathrm{R}$ with $\mathrm{P}$ sampling pixels, as shown in Fig. 1.

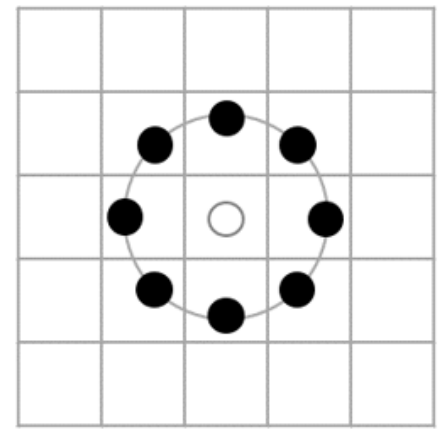

(a) $\operatorname{LBP}_{8}^{1}$

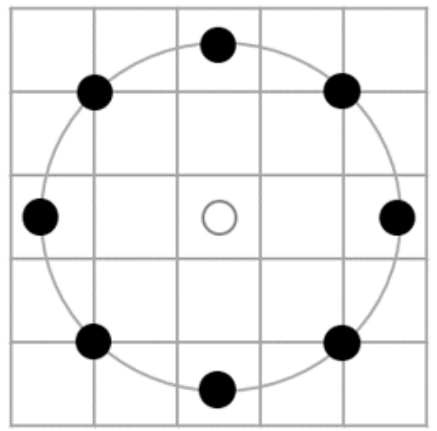

(b) $\mathrm{LBP}_{8}^{2}$

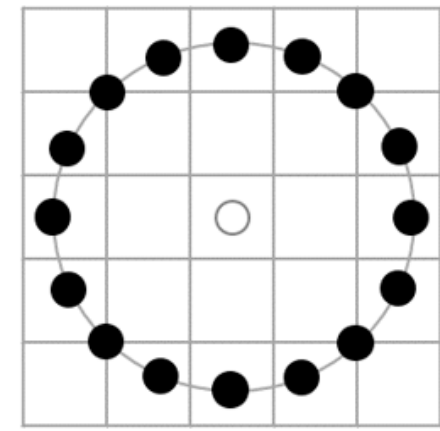

(c) $\mathrm{LBP}_{16}^{2}$

Fig. 1. Circularly symmetric neighbor sets for different $(\mathrm{P}, \mathrm{R})$

The formula is defined as follows:

$$
L B P_{P, R}=\sum_{p=0}^{P-1} s\left(g_{p}-g_{c}\right) 2^{p}, s(x)=\left\{\begin{array}{l}
1, x \geq 0 \\
0, x<0
\end{array} .\right.
$$

$\mathrm{R}$ is the radius of the neighborhood, $\mathrm{P}$ is the total number of pixels in circular area, $\mathrm{g}_{\mathrm{c}}$ is the gray value of the central pixel, $g_{p}$ is the value of its neighbors. In order to make the descriptor rotation invariant, a uniform rotation invariant LBP is proposed. The definition is as follows:

$$
L B P_{P, R}^{\text {riu } 2}= \begin{cases}\sum_{p=0}^{P-1} s\left(g_{p}-g_{c}\right) & \text { if } U\left(L B P_{P, R}\right) \leq 2 \\ P+1 & \text { otherwise }\end{cases}
$$

where

$$
\begin{aligned}
U\left(L B P_{P, R}\right) & =\left|s\left(g_{p-1}-g_{c}\right)-s\left(g_{0}-g_{c}\right)\right| \\
& +\sum_{p=1}^{P-1}\left|s\left(g_{p}-g_{c}\right)-s\left(g_{p-1}-g_{c}\right)\right|
\end{aligned}
$$

$\mathrm{U}$ measures the number of transitions from 0 to 1 or 1 to 0 in a binary sequence, $g_{0}$ is the value of the first pixel in the neighborhood of $g_{c}, g_{p-1}$ is the value of the p-1th pixel point in the neighborhood of $g_{c}$. (Binary sequence connected end to end).

For uniform rotation invariant LBP, all binary sequences which have more than 2 transitions are classified as the same mode. The mapping from $\operatorname{LBP}_{\mathrm{P}, \mathrm{R}}$ to $\mathrm{LBP}_{\mathrm{P}, \mathrm{R}}^{\mathrm{riu} 2}$, which has $\mathrm{P}+2$ distinct output values, can be implemented with a lookup table. 


\subsection{Multiple-Block LBP (MB-LBP)}

The LBP operator can describe the local texture information of the image. However, because of the features of localization, it easily can be affected by noise and not be robust enough due to the lack of a coarse-grained grasp of the overall image information. Fortunately, the MB-LBP algorithm can be used to overcome these deficiencies. In the calculation of the MB-LBP, the comparison between the pixel values of the LBP operator is replaced by the average gray value of the pixel block. MBS-LBP represents an LBP operator with a pixel block that measures SxS. The different pixel blocks represent different observation and particle size analyses, as shown in Fig. 2.

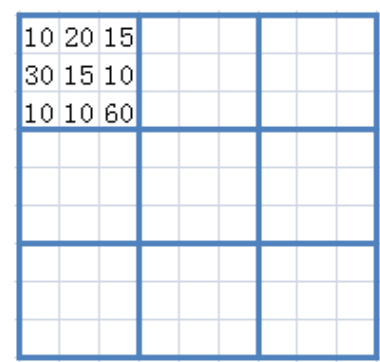

(a)

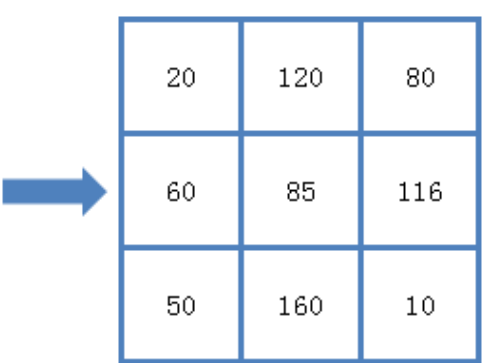

(b)

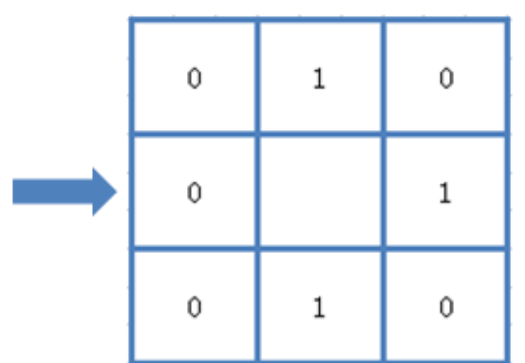

(c)

Fig. 2. (a) Each square in the fine line represents a pixel; (b) each square in the thick line represents a pixel block, and the value of the pixel block is the mean value of all pixels in the block; (c) calculated LBP after being divided into blocks

Formula of MB-LBP operator is defined as follows:

$$
M B-L B P=\sum_{n=0}^{7} s\left(i_{n}-i_{c}\right) 2^{n}, s(x)=\left\{\begin{array}{l}
1, x \geq 0 \\
0, x<0
\end{array} .\right.
$$

where $i_{n}$ is the mean value of the neighboring blocks, and $i_{c}$ is the mean value of the central pixel block. The total number of neighboring blocks is eight. Fig. 3 shows that, as the size $\mathbf{S}$ of the pixel block increases, the texture of image becomes coarser and tends to be stable, which shows that larger pixel blocks can help us grasp the coarse-grained information of an image.

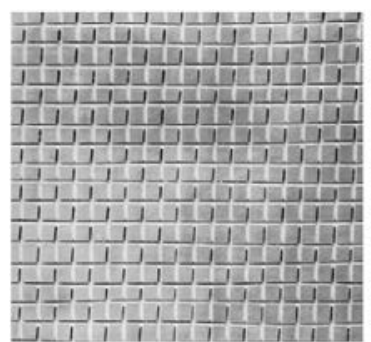

(a)

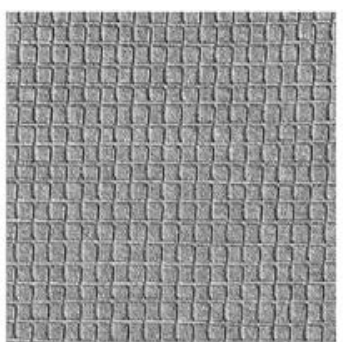

(b)

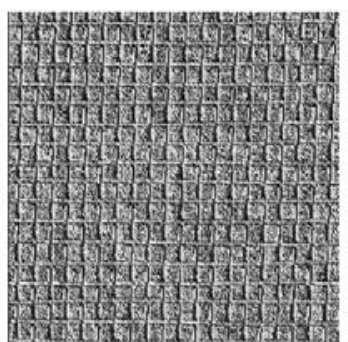

(c)

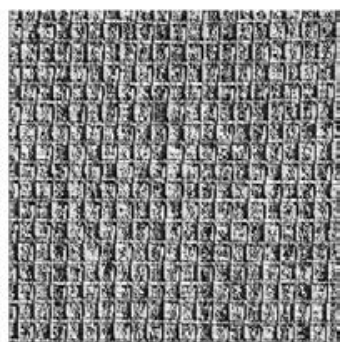

(d)

Fig. 3. Texture image after being filtered by $\mathrm{MB}_{S}-\mathrm{LBP}$ : (a) original; (b) after being filtered by $\mathrm{MB}_{1}-\mathrm{LBP}$; (c) after being filtered by $\mathrm{MB}_{2}-\mathrm{LBP}$; (d) after being filtered by $\mathrm{MB}_{3}-\mathrm{LBP}$ 


\subsection{A completed model of the local binary pattern (CLBP)}

The LBP operator proposed by Ojala et al. does a good job of texture classification. But, from the formula, we see that the result of the comparison between the center pixel and its neighboring pixels is either 0 or 1 , and it loses local difference information, which leads to two different textures being placed in the same classification. Fig. 4 shows an example of this. It is difficult to say that they have similar local structures.

\begin{tabular}{|c|c|c|}
\hline 60 & 120 & 80 \\
\hline 120 & 100 & 80 \\
\hline 30 & 150 & 160 \\
\hline
\end{tabular}

(a) LBP code: 01001101

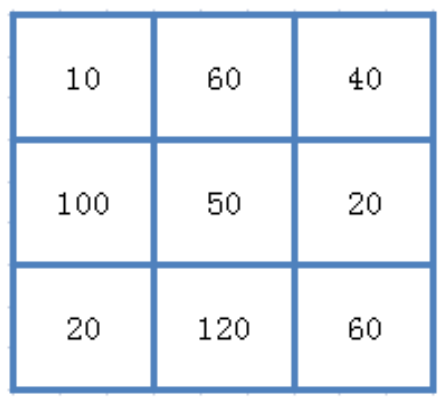

(b) LBP code: 01001101

Fig. 4. (a) and (b) have the same LBP code

In order to improve the ability to distinguish the local structures, Guo et al. proposed the completed LBP by combining CLBP_S, CLBP_M, and CLBP_C. The introduction of CLBP_M and CLBP C increases the distinguishable information of texture. The CLBP_S descriptor is equivalent to the original LBP descriptor, and the CLBP_M descriptor is the magnitude difference between center pixel and its neighboring pixels. Its formula is defined as follows:

$$
C L B P_{-} M_{P, R}=\sum_{i}^{P-1} s\left(m_{i}-c\right) 2^{i}, m_{i}=\left|g_{p}-g_{c}\right|, s(x)=\left\{\begin{array}{l}
1, x \geq 0 \\
0, x<0
\end{array}\right. \text {. }
$$

\section{Proposed method}

\subsection{Scalabe-Block CLBP(SB-CLBP)}

The CLBP proposed by Guo et al. adds extra distinguishable texture information, i.e., CLBP_M and CLBP_C, on the basis of LBP. Inspired by their work, we use slide window technique to calculate mean value of it and then calculate the CLBP of the mean image so that coarse-grained information can be reflected clearly. This technique is similar to that of multiple scales which have been widely used [22]. The coarse-grained information is added to the original CLBP, which is equivalent to the combination of local information and global information, resulting in further improvement in the texture classification ability of CLBP.

Fig. 5 shows the working principle of SB-CLBP. First, the CLBP_S, CLBP_M, and CLBP_C of the original image are calculated to obtain the mapping of CLBP, and then the statistical histogram is calculated. Second, the original image is divided into blocks, and the same calculation is carried out on them. The calculated CLBP histogram and CLBP histogram of the original image are combined and used for classification of texture.

Different slide window size represents different observation and analysis size. Therefore, we can use slide windows with different sizes and calculate their CLBP histogram to get different coarse-grained information. Their CLBP histograms are combined with the CLBP 
histogram of the original image to further improve the ability to classify the texture, as shown in Fig. 6.

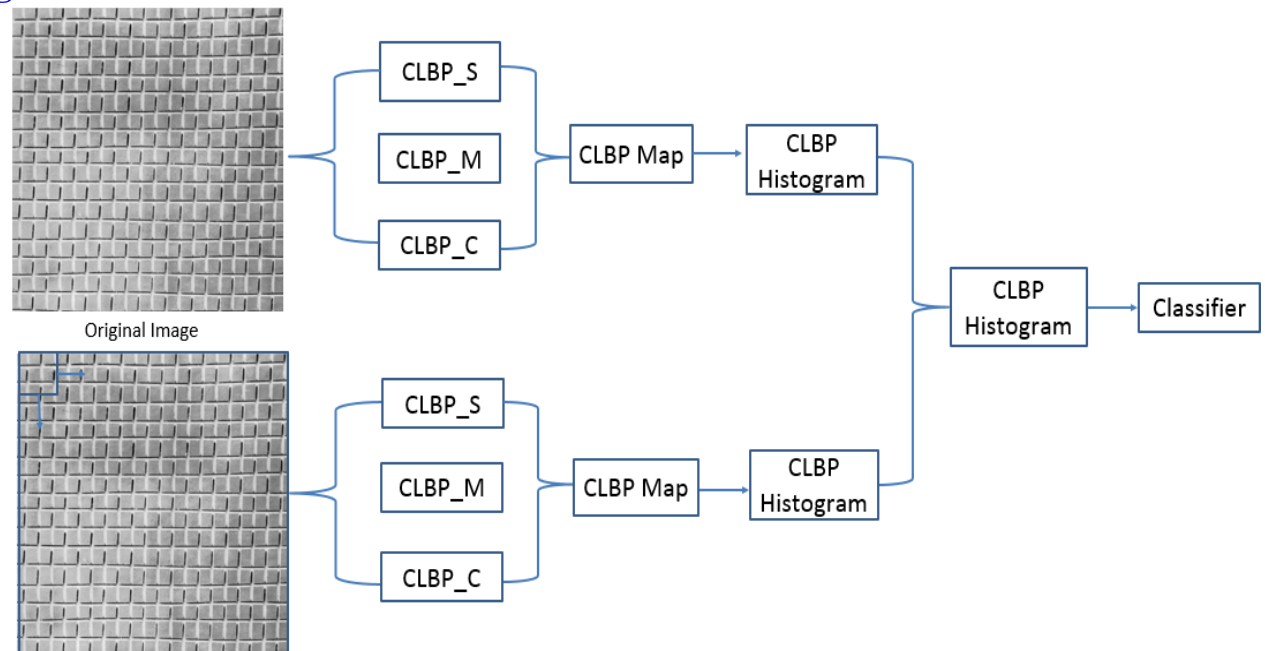

Scalable-Block Image

Fig. 5. The principle of SB-CLBP

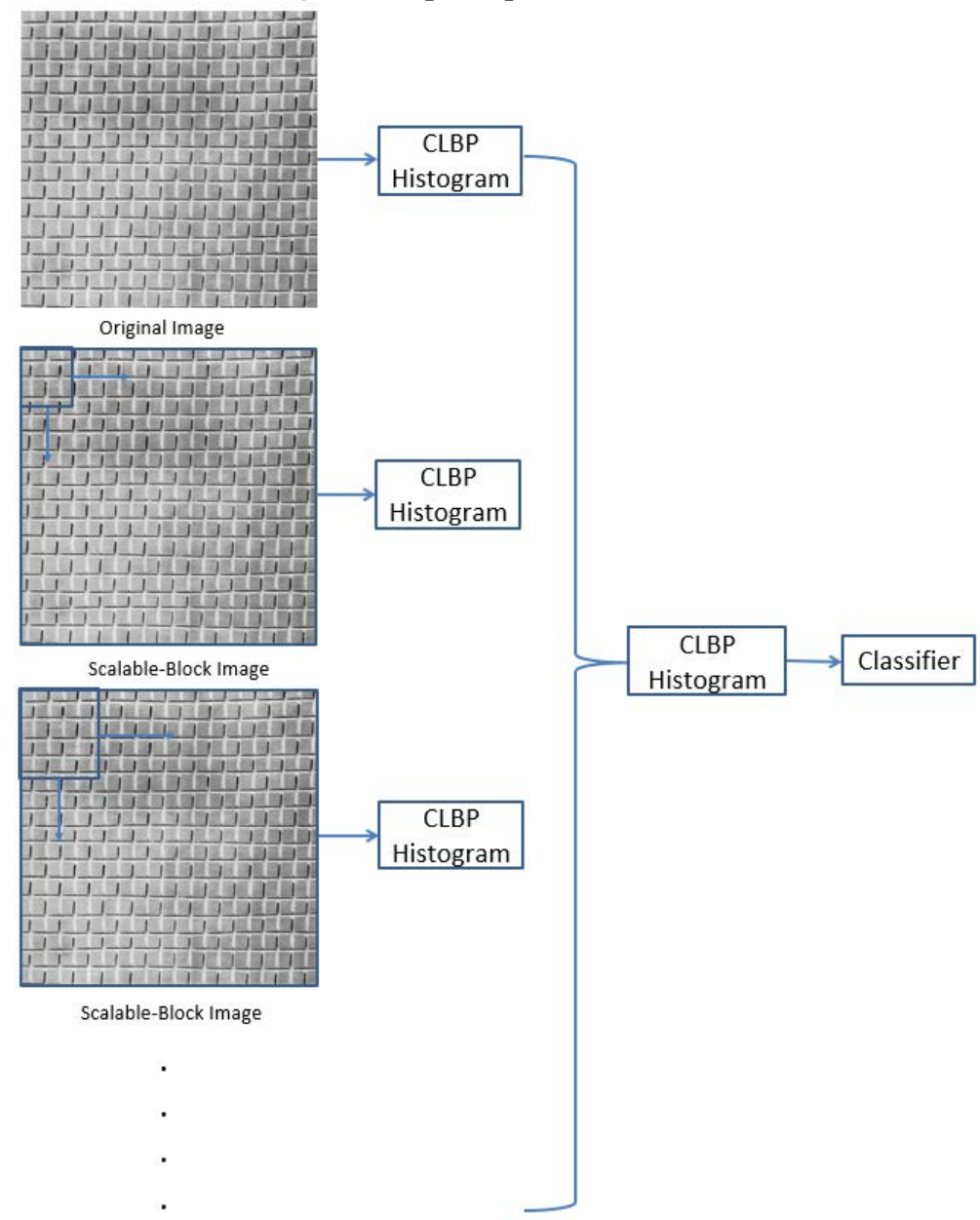

Fig. 6. A modified SB-CLBP 


\subsection{Our proposed scheme for texture classification}

The proposed scheme is divided into two parts: training and testing. The overall framework is shown in Fig 7. Numbers of samples from standard texture library are selected for training a module in the training part. The size of the selected image is of $128 * 128$ pixels. Classifier we choose is SVM or AdaBoost. Both SVM and AdaBoost have been extensively adopted in many applications. However, the training and incremental learning processes of AdaBoost are much slower than that of SVM [20]. Therefore, the SB-CLBP features of all the blocks are extracted to train the SVM classifier and then the trained model is used as the classifier. The flowchart of texture classification is shown as below. First, the CLBP histograms of kinds of texture images are calculated which contain CLBP histograms of original images and CLBP histograms of scalable-block images. On the other hand, the testing image follows the same schedule as the training part. In the end, it is classified by the trained SVM classifier.

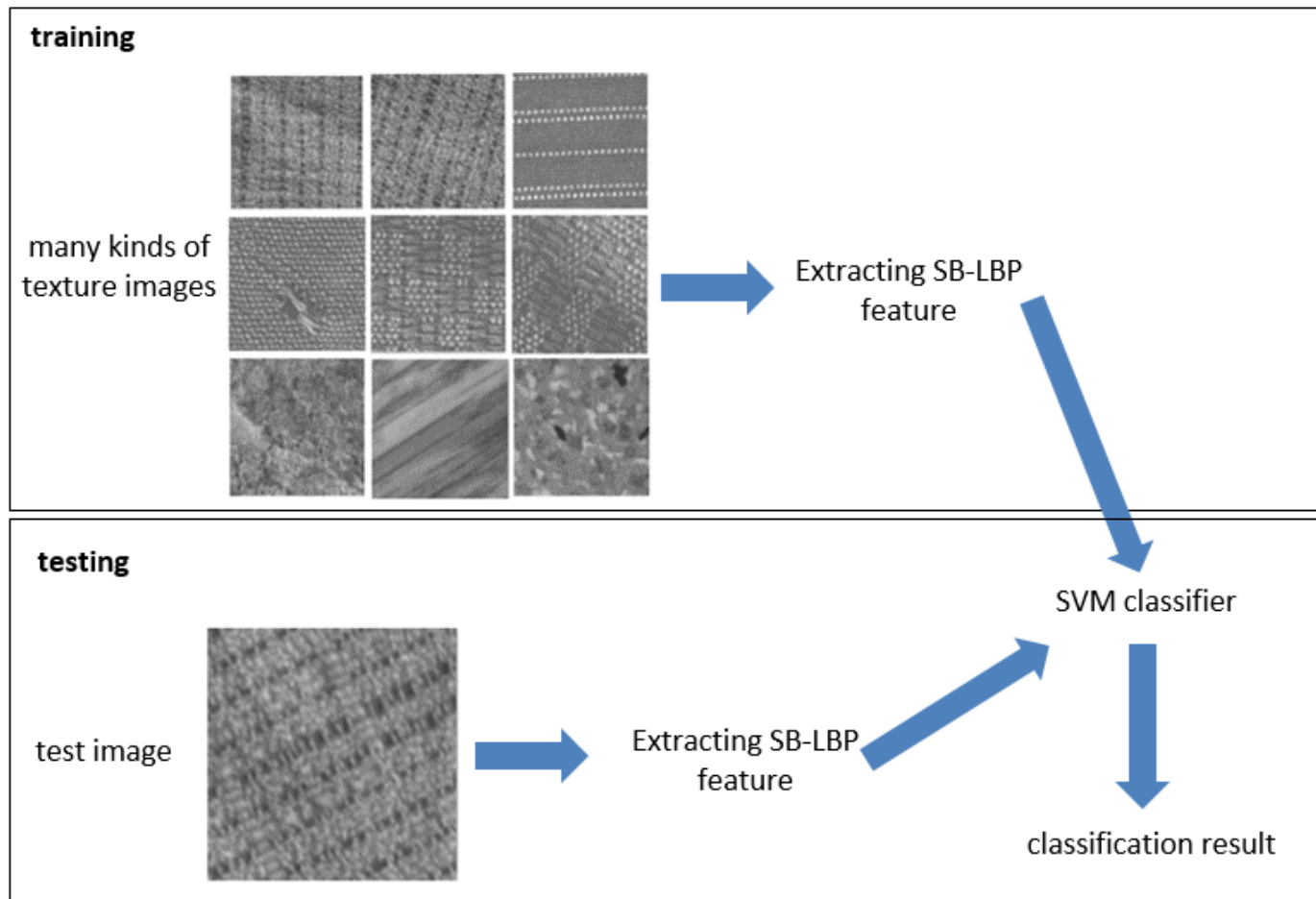

Fig. 7. Flowchart of texture classification

\section{Experimental Results and Analysis}

In order to verify the effectiveness of the proposed SB-CLBP texture feature extraction method, a series of experiments have been conducted on two representative texture databases, i.e., the Outex database [18] and the CUReT database [12]. We compared the proposed method with other methods, including CLBP, CJLBP [14], VZ_MR8 [13], VZ_Joint [15, 16], and URIG [17].

\subsection{Experimental results on the Outex database}

The Outex database, which includes the 24 classes of textures shown in Fig. 8, is used to demonstrate the significance of our proposed method. Two test suites in Outex are chosen in 
our experiment, i.e. Outex_TC_00010 (TC10) and Outex TC_00012 (TC12). They are collected under three different illuminants ("horizon", "inca”, and "t184") and nine different rotation angles $\left(0^{\circ}, 5^{\circ}, 10^{\circ}, 15^{\circ}, 30^{\circ}, 45^{\circ}, 60^{\circ}, 75^{\circ}\right.$ and $\left.90^{\circ}\right)$. There are 20 non-overlapping $128^{*} 128$ samples of texture in each class in order to fit every situation. For TC10, all textures in this test suite have the same illuminant, i.e., "inca". Samples of illuminant "inca" and angle $0^{\circ}$ in each class are used to train the classifier, and the other eight rotation angles with the same illumination are used as testing sets. Hence, there are $480(24 * 20)$ models for training and $3840(24 * 8 * 20)$ models for validation. For TC12, the texture classes are the same as TC10. The classifier is trained with the same training samples as TC10, but it is tested with all samples that are captured at all nine rotation angles with the different illuminants, i.e., t184 and horizon. Therefore, $480(24 * 20)$ models and $4320(24 * 20 * 9)$ validation samples are for each illuminance.
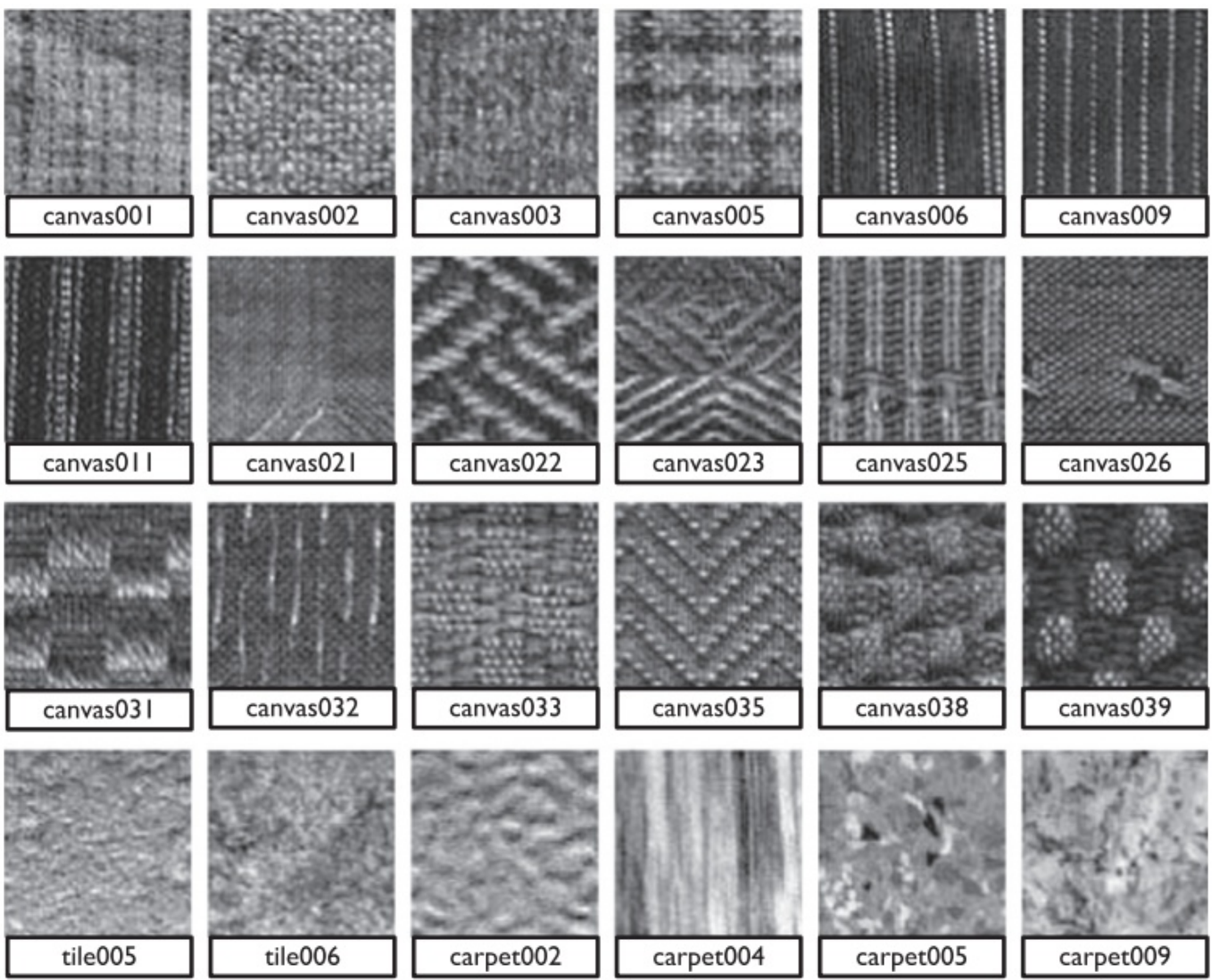

Fig. 8. 128*128 samples of the 24 different textures from Outex

Table 1 provides the experimental results under different experimental methods. The results of methods that are used as contrast are obtained directly from the literatures. By analyzing the results in Table 1, the following results are obtained:

First, for the proposed SB-CLBP_S descriptor, SB ${ }_{2+3+4}-$ CLBP_S $_{24,3}^{\text {riu2 }}(94.89 \%)$ and $\mathrm{SB}_{2+3}$-CLBP_S $\mathrm{S}_{24,3}^{\text {riu2 }}(93.82 \%)$ are improved much more than CLBP_S $\mathrm{S}_{24,3}^{\text {riu2 }}(86.96 \%)$.

Second, for the proposed SB-CLBP_M descriptor, SB ${ }_{2+3+4}-C L B P \_M_{24,3}^{\text {riu2 }}(93.64 \%)$ and $\mathrm{SB}_{2+3}-\mathrm{CLBP} \_\mathrm{M}_{24,3}^{\mathrm{riu} 2}(93.82 \%)$ are improved much more than CLBP_M $\mathrm{M}_{24,3}^{\mathrm{riu} 2}(85.11 \%)$. 
Third, for the combination of multiple descriptors, the method proposed in this paper also significantly improved the accuracy of classification. For example, the accuracy of $\mathrm{SB}_{3}$-CLBP_S $\mathrm{S}_{8,1}^{\text {riu2 }} / \mathrm{M}_{8,1}^{\text {riu2 }}(92.04 \%)$ is higher than CLBP_S $\mathrm{S}_{8,1}^{\text {riu2 }} / \mathrm{M}_{8,1}^{\text {riu2 }}(86.85 \%)$, and the $\mathrm{SB}_{2+3+4}$-CLBP_S $\mathrm{S}_{16,2}^{\text {riu2 }} \_\mathrm{M}_{16,2}^{\text {riu2 }} / \mathrm{C}(97.90 \%)$ descriptor is higher than the CLBP_S ${ }_{16,2}^{\text {riu2 }} \_\mathrm{M}_{16,2}^{\text {riu2 }} / \mathrm{C}$ (93.36\%) descriptor.

The classification accuracy of the descriptor, which is a combination of CLBP of two different block sizes, is higher than that of the single block descriptor. For instance, the $\mathrm{SB}_{2+3}-\mathrm{CLBP} \_\mathrm{S}_{\mathrm{P}, \mathrm{R}}^{\text {riu2 }} \_\mathrm{M}_{\mathrm{P}, \mathrm{R}}^{\text {riu2 }} / \mathrm{C}$ descriptor is better than $\mathrm{SB}_{3}-\mathrm{CLBP} \_S_{\mathrm{P}, \mathrm{R}}^{\text {riu2 }} \mathrm{M}_{\mathrm{P}, \mathrm{R}}^{\text {riu2 }} / \mathrm{C}$ descriptor. With the increase in the number of different block sizes, the accuracy of classification can be improved accordingly. For instance, $\mathrm{SB}_{2+3+4}$-CLBP_S ${ }_{\mathrm{P}, \mathrm{R}}^{\text {riu2 }} \_\mathrm{M}_{\mathrm{P}, \mathrm{R}}^{\text {riu2 }} / \mathrm{C}$ was better than $\mathrm{SB}_{2+3}-\mathrm{CLBP} \_S_{\mathrm{P}, \mathrm{R}}^{\text {riu2 }} \_\mathrm{M}_{\mathrm{P}, \mathrm{R}}^{\text {riu2 }} / \mathrm{C}$. For TC10, the $\mathrm{SB}_{2+3+4}-\mathrm{CLBP} \_\mathrm{S}_{16,2}^{\text {riu2 }} \_\mathrm{M}_{\mathrm{P}, \mathrm{R}}^{\text {riu2 }} / \mathrm{C}$ descriptor achieved the highest classification accuracy among the texture descriptors. But for TC12('t,' ' $h$ '), the accuracy of the CJLBP_SMC $C_{8,4,8+8,1,3,4}^{\text {riu2 }}$ descriptor is slightly higher than our proposed descriptor.

Table 1. The comparison of different experiments

\begin{tabular}{|c|c|c|c|c|c|c|c|c|c|c|c|c|}
\hline & \multicolumn{4}{|c|}{$(P, R)=(8,1)$} & \multicolumn{4}{|c|}{$(P, R)=(16,2)$} & \multicolumn{4}{|c|}{$(P, R)=(24,3)$} \\
\hline & \multirow[b]{2}{*}{ TC10 } & \multirow{2}{*}{\multicolumn{2}{|c|}{ TC12 }} & \multirow[b]{2}{*}{ Average } & \multirow[b]{2}{*}{ TC10 } & \multicolumn{2}{|c|}{ TC12 } & \multirow[b]{2}{*}{ Average } & \multirow[b]{2}{*}{ TC10 } & \multicolumn{2}{|c|}{ TC12 } & \multirow[b]{2}{*}{ Average } \\
\hline & & & "h" & & & "t $t^{\prime \prime}$ & "h" & & & "t $\mathrm{t}^{\prime \prime}$ & "h" & \\
\hline$C L B P_{-} S_{p \lambda}^{\Delta-2}$ & 84.81 & 65.46 & 63.68 & 71.31 & 89.40 & 82.26 & 75.20 & 82.28 & 95.07 & 85.04 & 80.78 & 86.96 \\
\hline$M B_{1}-C L B_{2} S_{2,2}^{\infty 21}$ & 92.50 & 81.11 & 75.32 & 82.98 & 94.66 & 89.03 & 84.91 & 89.53 & 98.07 & 91.69 & 86.60 & 92.12 \\
\hline$M B_{2+3}-C L B P_{-} S_{2 \ell-2}^{2}$ & 94.51 & 86.88 & 82.96 & 88.12 & 97.94 & 90.95 & 88.31 & 92.27 & 98.78 & 92.80 & 89.88 & 93.82 \\
\hline$M B_{2+1+4}-C L B P_{-} S_{p, 2}^{n-1}$ & 95.94 & 89.21 & 86.02 & 90.39 & 98.46 & 92.18 & 89.84 & 93.49 & 98.98 & 94.40 & 91.30 & 94.89 \\
\hline$C L B P_{-} M_{D, 2}^{r a 2}$ & 81.74 & 59.30 & 62.77 & 67.93 & 93.67 & 73.79 & 72.40 & 79.95 & 95.52 & 81.18 & 78.65 & 85.11 \\
\hline$M B_{s}-C L B P_{-} M_{\nu \lambda}^{\curvearrowright 2}$ & 87.84 & 69.54 & 71.78 & 76.39 & 97.63 & 84.07 & 86.06 & 89.25 & 98.72 & 87.78 & 85.44 & 90.65 \\
\hline$M B_{2+1}-C L B P_{-} M_{D, 2}^{2-2}$ & 91.22 & 74.31 & 75.02 & 80.18 & 98.83 & 84.51 & 86.94 & 90.09 & 99.19 & 89.77 & 88.06 & 92.34 \\
\hline$M B_{2+1+4}-C L B P_{-} M_{p, 2}^{r 21}$ & 91.59 & 75.28 & 76.92 & 81.26 & 99.24 & 87.36 & 89.42 & 92.01 & 99.27 & 91.90 & 89.75 & 93.64 \\
\hline$C L B P_{-} M_{p, 2}^{\prime 2,2} / C$ & 90.36 & 72.38 & 76.66 & 79.80 & 97.44 & 86.94 & 90.97 & 91.78 & 98.02 & 90.74 & 90.69 & 93.15 \\
\hline$M B_{3}-C L B P_{-} M_{p-\alpha}^{\infty 2 / C}$ & 93.02 & 80.79 & 83.17 & 85.66 & 99.06 & 91.16 & 93.84 & 94.69 & 99.06 & 92.99 & 93.36 & 95.14 \\
\hline$M B_{2+3}-C L B P_{-} M_{P, 2}^{2-2} / C$ & 94.43 & 83.50 & 86.34 & 88.09 & 99.45 & 92.85 & 94.51 & 95.60 & 99.27 & 93.98 & 93.82 & 95.69 \\
\hline$M B_{2+3+4}-C L B P_{-} M_{P, 2}^{2-2} / C$ & 95.03 & 84.44 & 87.78 & 89.08 & 99.66 & 94.17 & 95.86 & 96.56 & 99.38 & 94.91 & 94.58 & 96.29 \\
\hline 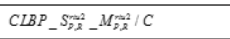 & 94.53 & 81.87 & 82.52 & 86.30 & 98.02 & 90.99 & 91.08 & 93.36 & 98.33 & 94.05 & 92.40 & 94.92 \\
\hline$M B_{s}-C L B P_{-} S_{p \ell-}^{\infty} M_{2, \lambda}^{-2} / C$ & 97.06 & 87.89 & 88.73 & 91.23 & 99.45 & 94.88 & 95.02 & 96.45 & 99.43 & 95.79 & 94.42 & 96.55 \\
\hline$M B_{2+3}-C L B P_{-} S_{p, 2}^{-2}-M_{\partial, 2}^{\infty 2} / C$ & 97.63 & 90.44 & 90.63 & 92.90 & 99.84 & 96.00 & 95.86 & 97.23 & 99.77 & 96.64 & 95.46 & 97.29 \\
\hline$M B_{2+1+4}-C L B P_{-} S_{p, d}^{\infty 21} M_{p, d}^{\infty 2} / C$ & 98.15 & 91.99 & 92.59 & 94.24 & 99.87 & 96.99 & 96.85 & 97.90 & 99.79 & 97.48 & 95.90 & 97.72 \\
\hline 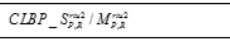 & 94.66 & 82.75 & 83.14 & 86.85 & 97.89 & 90.55 & 91.11 & 93.18 & 99.32 & 93.58 & 93.35 & 95.41 \\
\hline 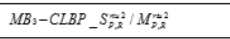 & 97.34 & 89.03 & 89.75 & 92.04 & 99.45 & 95.16 & 94.44 & 96.35 & 99.74 & 96.13 & 94.84 & 96.90 \\
\hline$M B_{2+1}-C L B P_{-} S_{P, \ell}^{-2} / M_{P, 2}^{-2}$ & 98.15 & 92.04 & 92.34 & 94.18 & 99.69 & 95.79 & 95.07 & 96.85 & 99.71 & 96.46 & 95.28 & 97.15 \\
\hline$M B_{2+3+4}-C L B P_{-} S_{P, 2}^{\infty-2} / M_{P, \Omega}^{\infty 2}$ & 98.59 & 93.54 & 93.96 & 95.36 & 99.77 & 96.62 & 95.69 & 97.36 & 99.77 & 97.20 & 95.81 & 97.59 \\
\hline$C L B P_{-} S_{\partial \lambda}^{S-2} / M_{\partial \lambda}^{\prime 2} / C$ & 96.56 & 90.30 & 92.29 & 93.05 & 98.72 & 93.54 & 93.91 & 95.39 & 98.93 & 95.32 & 94.53 & 96.26 \\
\hline 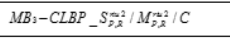 & 98.15 & 92.78 & 94.79 & 95.24 & 99.19 & 95.81 & 94.72 & 96.57 & 99.14 & 96.32 & 95.05 & 96.84 \\
\hline 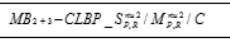 & 98.39 & 94.33 & 96.11 & 96.28 & 99.56 & 96.37 & 95.60 & 97.18 & 99.32 & 96.50 & 95.19 & 97.00 \\
\hline$M B_{2+3+4}-C L B P_{-} S_{p, d}^{s-2} / M_{2, d}^{\infty-2} / C$ & 98.57 & 94.88 & 96.44 & 96.63 & 99.61 & 96.81 & 95.58 & 97.33 & 99.32 & 96.60 & 95.23 & 97.05 \\
\hline$V Z_{-} M R 8$ & \multicolumn{12}{|c|}{ 93. 59(TC10), 92.55(TC12, "t $\left.\mathrm{t}^{\prime \prime}\right), 92.82\left(\mathrm{TC} 12,{ }^{\prime \prime} \mathrm{h}^{\prime \prime}\right)$ (Average 92. 99) } \\
\hline$V Z_{-} J o$ int & \multicolumn{12}{|c|}{ 92. $00(\mathrm{TC} 10), 91.41\left(\mathrm{TC} 12,{ }^{\prime \prime} \mathrm{t}^{\prime \prime}\right), 92.06\left(\mathrm{TC} 12,{ }^{\prime \prime} \mathrm{h}\right.$ ) (Average 91.82$)$} \\
\hline 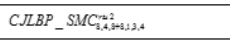 & \multicolumn{12}{|c|}{ 99. $77(\mathrm{TC} 10), 98.59\left(\mathrm{TC1} 2,{ }^{\prime \prime} \mathrm{t}^{\prime \prime}\right), 98.68\left(\mathrm{TC1},{ }^{\prime} \mathrm{h}^{\prime \prime}\right.$ ) (Average 99.01) } \\
\hline$U R I G / M_{(2,20)}$ & \multicolumn{12}{|c|}{ 97.73(TC10), 91.69(TC12, "t" ), 95.28(TC12, "h") (Average 94. 90) } \\
\hline
\end{tabular}

\subsection{Experimental results using the CUReT database}

For the CUReT database, the same subset of images are used as the previous experiments in [12], and 61 classes of textures are acquired from different perspectives and illumination orientations (Fig. 9). Each of them had 92 samples. In the experiments, $\mathrm{N}(\mathrm{N}=46,23,12,6)$ images are randomly selected from each class for training and the remaining $92-\mathrm{N}$ ones for test set. Table 2 lists the results of our texture classification using our proposed descriptor and other descriptors for comparison. Note that the results of VZ_MR8, VZ_Joint, and CJLBP_SMC $C_{8,1,2+8,4,7+8,1,2,3}^{\text {riu2 }}$ are obtained directly from the literature. Through the analysis in Table 2, we obtained the following results: 
First, with the decrease in the number of training samples, the accuracy of the classification will decrease. For example, the accuracy of CLBP_S $\mathrm{S}_{8,1}^{\text {ruz }}$ in $\mathrm{N}=46$ was $80.63 \%$, but when $\mathrm{N}=6$, it is reduced to $58.70 \%$. Other descriptors produced similar results.

Second, similar to Table 1, the descriptor that is a combination of CLBP of different block sizes significantly improved the classification accuracy. For instance, the accuracy of $\mathrm{SB}_{3}-\mathrm{CLBP} \_\mathrm{S}_{8,1}^{\text {riu2 }}(91.49 \%)$ is higher than that of CLBP_S $\mathrm{S}_{8,1}^{\text {riu2 }}(80.63 \%)$.

Third, our algorithm also improved the accuracy for the combination of CLBP_S, CLBP_M and CLBP_C. For instance, when $\mathrm{N}=46$, the accuracy of $\mathrm{SB}_{3}-\mathrm{CLBP} \mathrm{S}_{8,1}^{\text {riu2 }} / \mathrm{M}_{8,1}^{\text {riu2 }}$ (96.16\%) is higher than that of CLBP_S $\mathrm{S}_{8,1}^{\text {ruw }} / \mathrm{M}_{8,1}^{\text {riu2 }}(93.52 \%)$, and $\mathrm{SB}_{3}-\mathrm{CLBP} \mathrm{S}_{8,1}^{\text {riu2 }} / \mathrm{M}_{8,1}^{\text {riu2 }} / \mathrm{C}$ (96.98\%) was better than CLBP_S $\mathrm{S}_{8,1}^{\text {ruz }} / \mathrm{M}_{8,1}^{\text {riu2 }} / \mathrm{C}(95.59 \%)$.

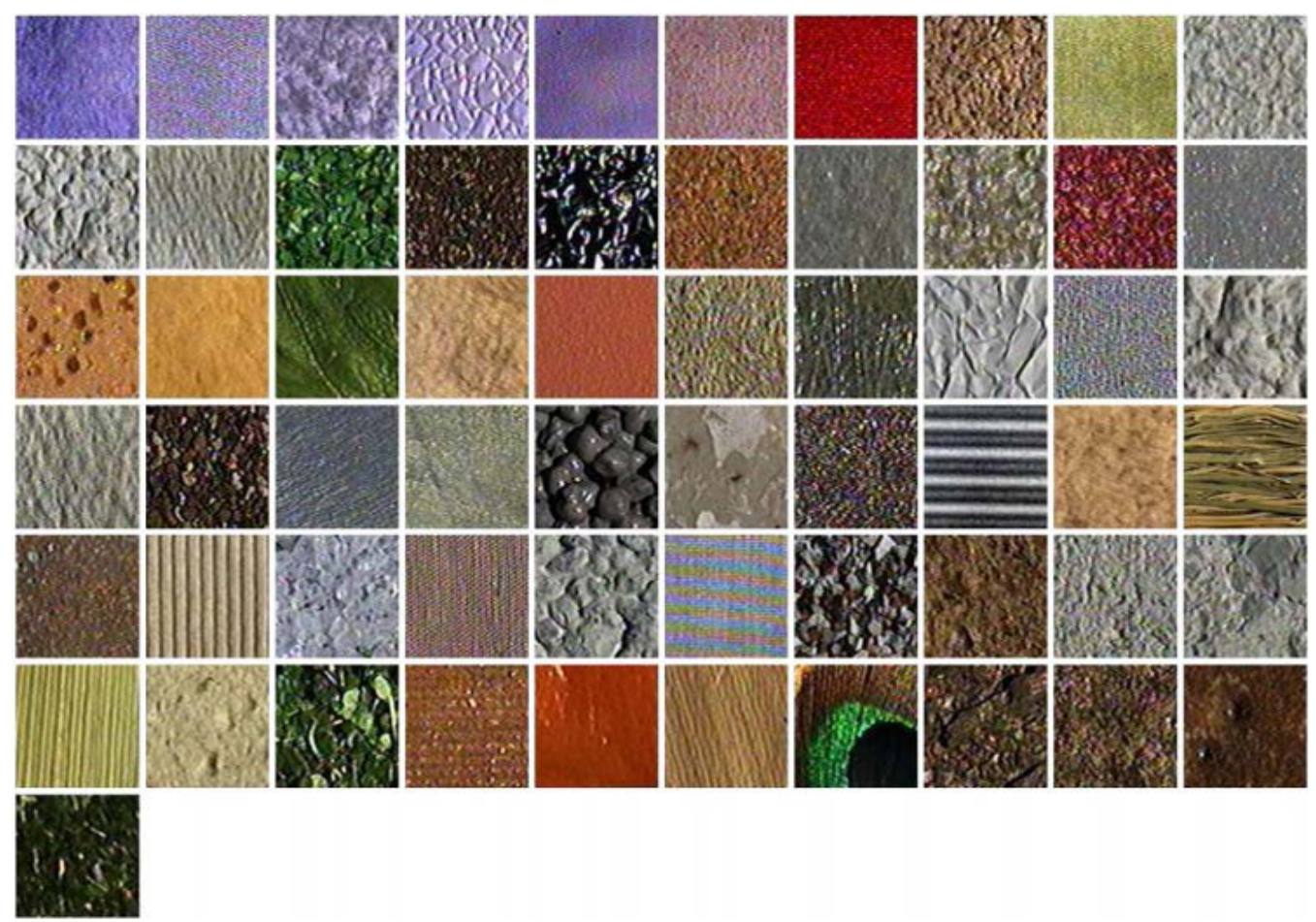

Fig. 9. Sixth textures in the CUReT dataset

The classification accuracy of the descriptor that was a combination of CLBP of two different block sizes was higher than that of the single block descriptor. For instance, when $\mathrm{N}$ $=46$, the accuracy of $\mathrm{SB}_{2+3}-\mathrm{CLBP} \_\mathrm{S}_{8,1}^{\text {riu2 }} / \mathrm{M}_{8,1}^{\text {riu2 }} / \mathrm{C}(97.33 \%)$ was higher than $\mathrm{SB}_{3}-\mathrm{CLBP} \mathrm{S}_{8,1}^{\text {riu2 }} / \mathrm{M}$ ${ }_{8,1}^{\text {riu2 }} / \mathrm{C}$. With an increasing the number of different block sizes, the accuracy of classification can be improved accordingly. For instance, when $N=46$, the accuracy of $\mathrm{SB}_{2+3+4}-\mathrm{CLBP} \_\mathrm{S}_{8,1}^{\text {riu2 }}$ /Mriu2 8,1/C (97.51\%) was higher than $\mathrm{SB}_{2+3} \mathrm{CLBP}_{-} \mathrm{S}_{8,1}^{\text {riu2 }} / \mathrm{M}_{8,1}^{\text {riu2 }} / \mathrm{C}(97.33 \%)$, and $\mathrm{SB}_{2+3+4+5}-\mathrm{CLBP} \mathrm{S}_{8,1}^{\text {riu2 }} / \mathrm{M}_{8,1}^{\text {riu2 }} / \mathrm{C}(97.76 \%)$ was higher than $\mathrm{SB}_{2+3+4}-\mathrm{CLBP}-\mathrm{S}_{8,1}^{\text {riu2 }} / \mathrm{M}_{8,1}^{\text {riu2 }} / \mathrm{C}(97.51 \%)$. 
Table 2. The comparison results for CUReT datasets

\begin{tabular}{|c|c|c|c|c|c|c|c|c|c|c|c|c|}
\hline \multirow{2}{*}{$\mathbb{N}$} & \multicolumn{4}{|c|}{$(P, R)=(8,1)$} & \multicolumn{4}{|c|}{$(P, R)=(16,2)$} & \multicolumn{4}{|c|}{$(P, R)=(24,3)$} \\
\hline & 46 & 23 & 12 & 6 & 46 & 23 & 12 & 6 & 46 & 23 & 12 & 6 \\
\hline$C L B P_{-} S_{\dot{P}, \overrightarrow{2}}$ & 80.63 & 74.81 & 67.84 & 58.70 & 86.37 & 81.05 & 74.62 & 66.17 & 86.37 & 81.21 & 74.71 & 66.55 \\
\hline$M B_{1}-C L B P_{-} S_{D 2}^{\infty 2}$ & 91.49 & 86.61 & 79.87 & 70.61 & 92.42 & 87.71 & 81.57 & 72.70 & 92.57 & 88.09 & 82.08 & 74.00 \\
\hline$M B_{1+3}-C L B P_{-} S_{P, 2}^{\infty-2}$ & 93.10 & 88.56 & 82.47 & 73.64 & 94.02 & 89.72 & 83.83 & 75.58 & 93.79 & 89.58 & 83.95 & 75.88 \\
\hline$M B_{2+3+4}-C L B P_{-} S_{p, 2}^{-2}$ & 94.40 & 90.22 & 84.52 & 75.77 & 94.67 & 90.74 & 85.17 & 77.03 & 94.40 & 90.22 & 84.52 & 75.77 \\
\hline$M B_{2+3+4+5-C L B P_{-}} S_{2 \lambda}^{2-2}$ & 95.36 & 91.44 & 85.76 & 77.46 & 94.87 & 91.49 & 86.08 & 77.97 & 94.98 & 91.31 & 85.84 & 77.74 \\
\hline$C L B P_{-} M_{p: 2}^{2-2}$ & 75.20 & 67.96 & 60.27 & 51.49 & 85.48 & 79.01 & 71.24 & 61.59 & 82.16 & 76.23 & 69.22 & 60.45 \\
\hline$M B_{3}-C L B P_{-} M_{2, \lambda}^{-2}$ & 86.67 & 79.71 & 71.52 & 61.62 & 90.84 & 85.02 & 77.31 & 67.45 & 90.85 & 85.04 & 77.29 & 67.40 \\
\hline$M B_{2+3}-C L B P_{-} M_{2, d}^{-2}$ & 88.77 & 82.02 & 74.15 & 64.07 & 93.19 & 88.03 & 80.79 & 70.95 & 92.65 & 87.32 & 80.35 & 70.65 \\
\hline$M B_{2+3+4}-C L B P_{-} M_{D, 2}^{-2}$ & 90.38 & 83.94 & 75.92 & 65.77 & 93.96 & 89.26 & 82.32 & 72.63 & 93.47 & 88.44 & 81.43 & 71.91 \\
\hline$M B_{2+3+4+\mathrm{s}}-C L B P_{-} M_{2, \lambda}^{2+2}$ & 91.29 & 85.26 & 77.48 & 67.30 & 94.30 & 90.08 & 83.18 & 73.78 & 93.95 & 89.08 & 82.24 & 72.37 \\
\hline$C L B P_{-} M_{\partial, \lambda}^{2-2} / C$ & 83.26 & 75.58 & 66.91 & 56.45 & 91.42 & 85.73 & 78.05 & 68.14 & 89.48 & 83.54 & 75.96 & 66.41 \\
\hline$M B_{1}-C L B P_{-} M_{p \perp}^{\infty 2} / C$ & 89.80 & 82.85 & 74.06 & 62.93 & 93.69 & 88.33 & 80.61 & 70.14 & 93.91 & 88.67 & 81.53 & 71.25 \\
\hline$M B_{2+3}-C L B P_{-} M_{p, 2}^{r-2} / C$ & 91.60 & 84.87 & 76.48 & 65.24 & 95.14 & 90.44 & 83.21 & 73.10 & 95.08 & 90.67 & 83.65 & 73.53 \\
\hline$M B_{2+3+4}-C L B P_{-} M_{p \alpha}^{-2} / C$ & 92.79 & 86.41 & 77.90 & 66.90 & 95.66 & 91.28 & 84.21 & 74.14 & 95.49 & 91.15 & 84.23 & 74.67 \\
\hline 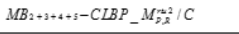 & 93.32 & 87.27 & 78.92 & 68.04 & 95.64 & 91.80 & 84.96 & 74.84 & 95.77 & 91.49 & 84.83 & 75.10 \\
\hline 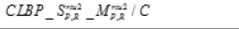 & 90.34 & 84.52 & 76.42 & 66.31 & 93.87 & 89.05 & 82.46 & 72.51 & 93.22 & 88.37 & 81.44 & 72.01 \\
\hline 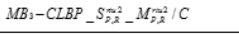 & 94.74 & 89.66 & 82.34 & 71.77 & 95.57 & 91.34 & 84.68 & 74.88 & 95.79 & 91.74 & 85.29 & 76.08 \\
\hline$M B_{2+3}-C L B P_{-} S_{p, 2}^{\infty 2}-M_{p-\alpha}^{\infty 2} / C$ & 95.32 & 90.98 & 83.79 & 73.66 & 96.36 & 92.65 & 86.49 & 77.15 & 96.52 & 92.69 & 86.84 & 77.65 \\
\hline 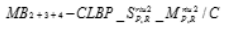 & 96.06 & 91.86 & 84.92 & 74.97 & 96.80 & 93.18 & 87.34 & 78.34 & 96.87 & 93.33 & 87.50 & 78.07 \\
\hline 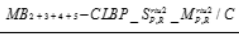 & 96.54 & 92.49 & 85.80 & 75.97 & 96.79 & 93.72 & 87.91 & 78.94 & 97.09 & 93.67 & 87.81 & 79.02 \\
\hline$C L B P_{-} S_{p, 2}^{-2} / M_{p, 2}^{2-2}$ & 93.52 & 88.67 & 81.95 & 72.30 & 94.45 & 90.40 & 84.17 & 75.39 & 93.63 & 89.14 & 82.47 & 73.26 \\
\hline 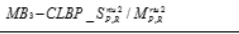 & 96.16 & 92.16 & 86.20 & 77.05 & 95.94 & 92.22 & 86.50 & 77.78 & 95.85 & 92.18 & 86.38 & 77.46 \\
\hline$M B_{2+3}-C L B P_{-} S_{2, \Omega}^{\infty-1} / M_{D, 2}^{-1}$ & 96.63 & 93.19 & 87.38 & 78.27 & 96.76 & 93.30 & 87.96 & 79.32 & 96.28 & 92.73 & 87.16 & 78.67 \\
\hline$M B_{2+3+4}-C L B P_{-} S_{p, 8}^{n-2} / M_{p, 2}^{\infty-2}$ & 97.06 & 93.65 & 88.17 & 79.32 & 97.19 & 93.89 & 88.77 & 80.13 & 96.62 & 93.26 & 87.82 & 79.00 \\
\hline$M B_{2+3+4+3-C L B P_{-}} S_{p d}^{-2} / M_{p, 2}^{-2}$ & 97.36 & 94.03 & 88.80 & 80.06 & 97.11 & 94.36 & 89.10 & 80.77 & 96.91 & 93.62 & 88.09 & 79.82 \\
\hline$C L B P_{-} S_{p, d}^{-2} / M_{D, \lambda}^{-2} / C$ & 95.59 & 91.35 & 84.92 & 74.80 & 95.86 & 92.13 & 86.15 & 77.04 & 94.74 & 90.33 & 83.82 & 74.46 \\
\hline 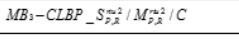 & 96.98 & 93.43 & 87.16 & 77.86 & 96.75 & 93.31 & 87.59 & 78.30 & 96.56 & 93.11 & 87.21 & 78.29 \\
\hline$M B_{2+1}-C L B P_{-} S_{-, \Omega}^{\infty-2} / M_{D, \Omega}^{\infty-2} / C$ & 97.33 & 94.07 & 88.20 & 79.03 & 97.21 & 94.07 & 88.62 & 79.78 & 96.93 & 93.64 & 88. 11 & 79.23 \\
\hline$M B_{2+3+4}-C L B P_{-} S_{p, d}^{-2} / M_{R, d}^{20.2} / C$ & 97.51 & 94.31 & 88.72 & 79.59 & 97.44 & 94.45 & 89.21 & 80.27 & 97.06 & 93.89 & 88.27 & 79.39 \\
\hline$M B_{2+3+4+3}-C L B P_{-} S_{D, 2}^{n-2} / M_{D, d}^{r a-1} / C$ & 97.76 & 94.76 & 89.30 & 80.39 & 97.29 & 94.60 & 89.37 & 80.64 & 96.70 & 93.47 & 88.04 & 79.29 \\
\hline$V Z_{-} M R 8$ & & & & & $.79(46)$ & $5.03(23$ & $90.48(1)$ & , 82.90 & & & & \\
\hline$V Z_{-} J o$ int & & & & & $.66(46)$ & $4.58(23$ & $89.40(12$ &, 81.06 & & & & \\
\hline 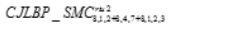 & & & & & $.51(46)$ & $4.75(23$ & 89. $73(1$ & , 79.89 & & & & \\
\hline
\end{tabular}

\subsection{Analysis of experimental results}

As stated above, the LBP descriptor only extracts the sign value of the pixel while the CLBP descriptor adds both magnitude and center information for feature extraction of texture. Therefore, the CLBP descriptor is more accuracy than the LBP. Also, the quality of feature extraction of texture mainly depends on the type of texture. Both gross and detailed information are related to the accuracy of feature extraction. Therefore, the feature extraction strategy based on the CLBP features of a scalable block size is implemented in this paper. Through experiments, we find some texture images are able to be classified and recognized correctly by extracting their SB-CLBP features while they fail only with LBP or CLBP feature as shown in Fig 10 and Fig 11. In Fig 10, the testing image with category 10 is wrongly recognized as category 16 because of their similar CLBP_S feature, i.e. LBP feature. However, they can be classified correctly when the CLBP_S features of multiple block size are extracted, such as the block sizes are set to 2, 3 and 4. Also, as shown in Fig 11, the more CLBP features with multiple block sizes are used, the more accuracy classification and recognition can be achieved. 

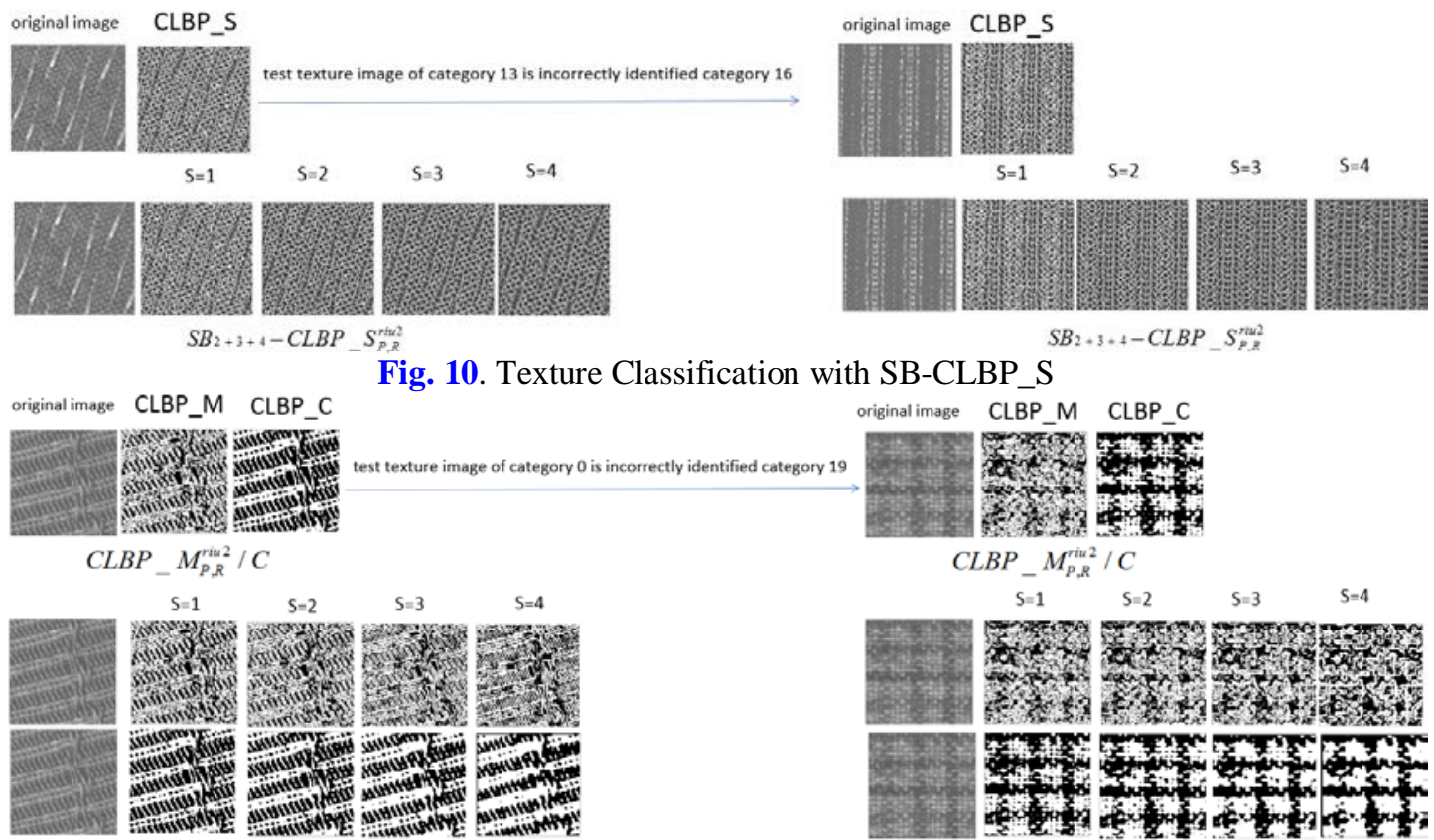

$S B_{2+3+4-C L B P} M_{P, R}^{r b 2} / C$

Fig. 11. Texture Classification with SB-CLBP_M/C

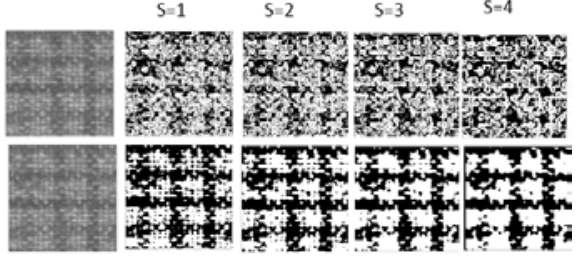

$S B_{2+3+4-C L B P} \quad M_{P, 2}^{r h 2} / C$

\section{Conclusion}

In this paper, a novel texture descriptor based on the CLBP features of a scalable block size is proposed for texture classification and recognition. SB-CLBP descriptor not only has advantages as other LBP descriptors, such as simple calculations, the invariance of the light and rotation angles, and the robustness of the noise, but also it extracts both coarse-grained and detailed features of a texture image and therefore, it has a higher classification and recognition accuracy. A complete strategy combining SB-CLBP feature extraction, machine leading and SVM has implemented in this paper. Experiments based on two standard libraries of texture classification have been completed and compared with the previous researches. The experimental results shows that the proposed scheme with the SB-CLBP descriptor is indeed a good approach in texture classification and recognition.

\section{References}

[1] Ojala, Timo, Matti Pietikainen, and Topi Maenpaa, "Multiresolution gray-scale and rotation invariant texture classification with local binary patterns," IEEE Transactions on pattern analysis and machine intelligence, vol. 24, no. 7, pp. 971-987, July, 2002.

Article (CrossRef Link)

[2] Ahonen, Timo, Abdenour Hadid, and Matti Pietikainen, "Face description with local binary patterns: Application to face recognition," IEEE transactions on pattern analysis and machine intelligence, vol. 28, no, 12, pp, 2037-2041, December, 2006. Article (CrossRef Link)

[3] Zhao, Guoying, and Matti Pietikainen, "Dynamic texture recognition using local binary patterns with an application to facial expressions," IEEE transactions on pattern analysis and machine intelligence, vol. 29, no. 6, June, 2007. Article (CrossRef Link)

[4] Nanni, Loris, and Alessandra Lumini, "RegionBoost learning for 2D+3D based face recognition,” Pattern Recognition Letters, vol. 28, no. 15, pp. 2063-2070, November, 2007. 
Article (CrossRef Link)

[5] Shih, Frank Y., and Chao-Fa Chuang, "Automatic extraction of head and face boundaries and facial features,” Information Sciences, vol. 158, pp. 117-130, January, 2004.

Article (CrossRef Link)

[6] Liao, Shu, Max WK Law, and Albert CS Chung, "Dominant local binary patterns for texture classification,” IEEE transactions on image processing, vol. 18, no. 5, pp. 1107-1118, May 2009. Article (CrossRef Link)

[7] Guo, Zhenhua, Lei Zhang, and David Zhang, "Rotation invariant texture classification using LBP variance (LBPV) with global matching,” Pattern recognition, vol. 43, no. 3, pp. 706-719, March, 2010. Article (CrossRef Link)

[8] Liu, Li, et al, "BRINT: binary rotation invariant and noise tolerant texture classification," IEEE Transactions on Image Processing, vol. 23, no. 7, July, 2014. Article (CrossRef Link)

[9] Huang, Di, et al, "3-d face recognition using elbp-based facial description and local feature hybrid matching," IEEE Transactions on Information Forensics and Security, vol. 7, no. 5, pp. 1551-1565, Oct, 2012. Article (CrossRef Link)

[10] Iakovidis, Dimitris K., Eystratios G. Keramidas, and Dimitris Maroulis, "Fuzzy local binary patterns for ultrasound texture characterization," in Proc. of International Conference Image Analysis and Recognition, vol. 5112, pp. 750-759, June, 2008. Article (CrossRef Link)

[11] Guo, Zhenhua, Lei Zhang, and David Zhang, "A completed modeling of local binary pattern operator for texture classification," IEEE Transactions on Image Processing, vol. 19, no. 6, pp. 1657-1663, June 2010. Article (CrossRef Link)

[12] Dana, Kristin J., et al, "Reflectance and texture of real-world surfaces," ACM Transactions on Graphics (TOG), vol. 18, no. 1, pp. 1-34, January 1999. Article (CrossRef Link)

[13] Varma, Manik, and Andrew Zisserman, "A statistical approach to texture classification from single images," International Journal of Computer Vision, vol. 62, no. 1, pp. 61-81, April 2005. Article (CrossRef Link)

[14] Wu, Xiaosheng, and Junding Sun, "Joint-scale LBP: a new feature descriptor for texture classification,” The Visual Computer, pp. 1-13, 2015. Article (CrossRef Link)

[15] Varma, Manik, and Andrew Zisserman, "Texture classification: Are filter banks necessary?,” in Proc. of Computer vision and pattern recognition, 2003, Proceedings. 2003 IEEE computer society conference on, vol. 2, pp. II-691, June, 2003. Article (CrossRef Link)

[16] Varma, Manik, and Andrew Zisserman, "A statistical approach to material classification using image patch exemplars," IEEE transactions on pattern analysis and machine intelligence, vol. 31, no. 11, pp. 2032-2047, November, 2009. Article (CrossRef Link)

[17] Zhao, Wenteng, Zongqing Lu, and Qingmin Liao, "Texture classification using uniform rotation invariant gradient," in Proc. of Image Processing (ICIP), 2015 IEEE International Conference on, pp. 3650-3654, September, 2015. Article (CrossRef Link)

[18] Ojala, Timo, et al, "Outex-new framework for empirical evaluation of texture analysis algorithms," in Proc. of Pattern Recognition, 2002. Proceedings. 16th International Conference on, vol. 1, pp. 701-706, 2002. Article (CrossRef Link)

[19] Heikkilä, Marko, Matti Pietikäinen, and Cordelia Schmid, "Description of interest regions with local binary patterns,” Pattern recognition, vol. 42, no. 3, pp. 425-436, March, 2009. Article (CrossRef Link)

[20] Wen, Xuezhi, et al, “A rapid learning algorithm for vehicle classification,” Information Sciences, vol. 295, no. 1, pp. 395-406, February, 2015. Article (CrossRef Link)

[21] Qin, Chuan, et al, "A novel image hashing scheme with perceptual robustness using block truncation coding," Information Sciences, vol. 361-362, pp. 84-99, September, 2016.

Article (CrossRef Link)

[22] Yuan, Chengsheng, Xingming Sun, and Rui Lv, "Fingerprint liveness detection based on multi-scale LPQ and PCA,” China Communications, vol. 13, no. 7, pp. 60-65, July, 2016. Article (CrossRef Link) 

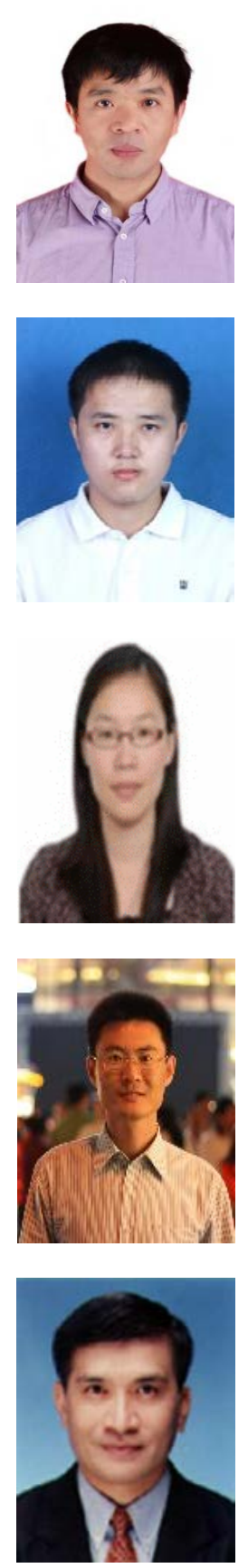

Jianjun Li received the B.Sc. degree in information engineering from Xi'an University of Electronic Science and Technology, Xi'an, China, and the M.Sc. and $\mathrm{Ph} . \mathrm{D}$ degrees in electrical and computer from The University of Western Ontario and University of Windsor, Canada separately. He is currently working at HangZhou Dianzi University as a chair professor. His research interests include micro-electronics, audio, video and image processing algorithms and implementation.

Susu Fan is in his third year of the master program at Hangzhou Dianzi University, Zhejiang, China, in 2016. His major is Computer Technology. He received his BS degree in Computer Science and Technology in 2012 in Nanchang Institute of Technology, Nanchang, China. His research interests include object detection, computer vision and machine learning.

Zhi-Hui Wang was born in Inner Mongolia Province, China, in 1982. She received a BS degree in software engineering in 2004 from the North Eastern University, Shenyang, China, and an MS degree in software engineering in 2007 from the Dalian University of Technology, Dalian, China. She received her PhD degree in computer software and theory from the Dalian University of Technology, Dalian, China. Her research interests include information hiding and image processing

Haojie Li is a Full Professor in the School of Software, Dalian University of Technology. He received the B.E. and the Ph. D. degrees from Nankai University, Tianjin and the Institute of Computing Technology, Chinese Academy of Sciences, Beijing, in 1996 and 2007 respectively. From 2007 to 2009, he was a Research Fellow in the School of Computing, National University of Singapore. He is a member of IEEE and ACM. His research interests include computer vision, social media computing and multimedia information retrieval.

Chin-Chen Chang received his Ph.D. degree in computer engineering from National Chiao Tung University. His first degree is Bachelor of Science in Applied Mathematics and master degree is Master of Science in computer and decision sciences. His current title is Chair Professor in Department of Information Engineering and Computer Science, Feng Chia University, from Feb. 2005. Prior to joining Feng Chia University, Professor Chang was an associate professor in Chiao Tung University, professor in National Chung Hsing University, chair professor in National Chung Cheng University. He had also been Visiting Researcher and Visiting Scientist to Tokyo University and Kyoto University, Japan.He is currently a Fellow of IEEE and a Fellow of IEE, UK. His current research interests include database design, computer cryptography, image compression and data structures. 